\title{
A new hope? Experiences of accessibility of services in deaf and hard-of-hearing audiences post-digital television switchover
}

Leighton Evans, Maynooth University

Yan Wu, Swansea University

Elain Price, Swansea University

\begin{abstract}
One promise of the switchover from analogue to digital television was new accessibility solutions. In the case of deaf and hard-of-hearing audiences who rely on subtitling for comprehension, the digital switchover makes it possible for greater provision of subtitling or improvements in accuracy. Utilizing quantitative data from a questionnaire completed by 339 participants with varying degrees of hearing difficulty in Wales, this article assesses perceptions of subtitling pre- and post-digital switch. A within-group comparison across age groups is also used to assess whether improvements in service are age defined. The results found that difficulties in accessing quality subtitling are still experienced by this audience post-digital switchover and that there are no significant differences in age in experiencing these difficulties. Knowledge of other digital services is subject to a significant difference in age, which indicates more work is required to inform older audience members of the affordances of digital television.
\end{abstract}

\section{Keywords}

affordances

barriers

deaf and hard-of-hearing audiences

digital television

interactive services

subtitling 
Wales

\section{Introduction}

Television in the United Kingdom has undergone a compulsory digital transition in stages from 2005. Begun in Ferryside, West Wales, the process of switchover has involved a migration to digital television (DTV) services through the use of Freeview services or satellite or cable services that provide DTV over non-signal broadcast means. Wales is United Kingdom's first DTV nation since the digital switchover was completed in March 2010 (DigitalUK 2012). This technological revolution brought in new forms of data dissemination that transformed not only the ways people acquire information from television but also the way people interact with television services, as well as creating more viewing choice. As Ford Ennals, CEO of DigitalUK claimed in 2006, the digitalization heralded a 'true golden age of television' (DigitalUK 2006). Wales' status as a testing ground for the digital switchover affords the possibility of researching the effects of the technological change on audience experience of television post-switchover. Accordingly, the research in this article was conducted between 2012 and 2013 (when the switchover was complete in Wales), and specifically concerned the effects of the digital switchover on deaf and hard-ofhearing audiences. Wales itself was an ideal site for research not only due to the early switchover date in relation to the rest of the United Kingdom. According to the most recent health survey, around one in seven adults in Wales (or 15 per cent) reported having difficulties with hearing (Welsh Government 2013). This health concern increased with age, affecting around one-third of senior citizens aged 65 years and over and affecting men more than women. By 2031, the projection for the total number of people in Wales with hearing loss will be more than 725,000 (Wales Mental Health in Primary Care n.d.).

While most attention in digital media and communication research concentrates on Internet-based communications, social media and convergence technologies and applications, 
the role of television in everyday life is still very important despite audience fragmentation and new media technologies diluting audience numbers. In the United Kingdom, the average time spent viewing television by adults is 3 hours 52 minutes a day - which is only a decline of 9 minutes from 2012, but is an increase in viewing of 10 minutes since 2004 (Ofcom 2014). Even with new devices and Internet-based communication, television maintains its audience and popularity. Research into the impact of digitalization of television reveals the political and commercial interdependence associated with the switchover (Starks 2007), the role of the public broadcasting in the switchover and the implication of such changes on public broadcasting service (Iosifidls 2005, 2007; Smith and Steemers 2007) and the second screen as an addition to the viewing experience (Lochrie and Coulton 2012). However, current research into DTV tends to focus either on macro-level issues such as public service broadcasting or (at an audience-member level) on technologically savvy 'early adopters' and tends to overlook the vulnerable social groups such as the audiences suffering from sensory impairment.

Action on Hearing Loss (2011) reports that there are more than 10 million adults in the United Kingdom with some form of hearing loss, or one in six of the population. It remains largely unclear how this community copes with the new digital media environment brought about by DTV. It is therefore this article's objective to assess the impact of digital switchover on the vulnerable social groups via a case study of deaf and hard-of-hearing audiences in Wales. We note that current regulating measures place emphasis more on quantity (e.g. the percentage of television programme subtitling) but less on quality, echoing the dominance of macro-level issues identified in research on DTV at the policy level regarding accessibility of services. Over emphasizing the quantity of subtitling could lead to overlooking other quality-related constraints (e.g. poor quality of sound in television programmes) faced by the deaf and hard-of-hearing audience. This research investigates the 
possibility of DTV being a form of assistive technology and whether it enables the deaf and hard-of-hearing community to have more access to information and services.

Brooks (2006) identified that people with sensory disabilities, like anyone else, need access to timely emergency warning information. Primary information sources, such as radio and television broadcasts, do not consistently serve the needs of deaf and hard-of-hearing people. When assessing DTV as a technology that addresses such accessibility issues, the primary consideration is how the technological platform provides affordances to the deaf and hard-of-hearing audience. The advent of DTV services was identified as one possible means of addressing this deficit, through the affordances (Gibson 1977; Norman 2013) offered by the digital platform. As Norman (2013: 11) argues, 'An affordance is a relationship between the properties of an object and the capabilities of the agent that determine just how the object could possibly be used'. DTV, it is argued, offers a set of affordances that differ from analogue television with regard to how the 'agent' can use that television. Here, 'affordance' is used to refer to the relationship among the technology, the environment and the user that allows the user to perform a particular action (i.e. watching, enjoying and using television to participate in social and cultural affairs). With regard to the deaf and hard-of-hearing audience, these affordances could result in overcoming barriers to watching television, understanding content and participating in audience experiences as well as being informed and entertained. This research specifically set out to investigate and understand how the additional affordances of the digital platform for television have, if at all, enabled deaf and hard-of-hearing audiences to access television services. In doing this, we identify whether historic barriers to access have been eradicated or minimized by the digital platform. Based on the study of deaf and hard-of-hearing audience members that we present here, we argue that problems with accessibility have persisted through the switch to DTV, and further work 
in policy and education needs to be done to utilize the digital platform to improve services for this audience.

\section{DTV as affordable technology: The importance of television subtitling and the technological platform}

The importance of television viewing itself is, foundationally, important to consider because various academic researchers have found that visual media (especially television) can alleviate the frustration and social exclusion in physical, mental and social domains for the deaf and hard-of-hearing community (Austin 1980; Austin and Myers 1984). Uncorrected or unaided hearing loss gives rise to poorer quality of life that is related to isolation, reduced social activity, and increased prevalence of symptoms of depression (Arlinger 2003). Inclusivity for deaf and hard-of-hearing audiences in mass media broadcasts is therefore critical for social inclusivity, psychological well-being and quality of life. Chia et al. (2007) reiterated an association between hearing impairment and health-related quality of life in an older population. Age and aging in this population is of critical salience; the deaf and hard-ofhearing audience is skewed in age profile towards older people, and following Van Dijk (2005) the notion of the 'digital divide' has a role to play in this research. 'Digital divide' refers to the exclusion of older persons from the use of digital media as a factor of familiarity, socialization and access. As inclusivity with regard to media provision is critical to quality of life, and as an initial research question (RQ1) in this article, the data will be used to assess if television is used more by older deaf and hard-of-hearing audiences than younger people of the same group. This question identifies the importance of television to this audience group, accounting for fragmentation and competition from other platforms.

It is important to understand what affordances offered by television are essential in allowing deaf and hard-of-hearing viewers to participate as an audience. While television may be a critical tool in including deaf and hard-of-hearing people in everyday life, the 
obvious barriers to inclusivity have detrimental effects on the use of the medium. SanchoAldridge and Davis (1993) investigated the impact of hearing impairment on television viewing in the United Kingdom. Hard-of-hearing viewers were found to report much greater difficulty watching programmes (mean difficulty rating $=32$ per cent) than elderly viewers with no reported hearing difficulty (mean difficulty rating $=10$ per cent), or those viewers generally who said they had no hearing problems (mean difficulty rating $=3$ per cent). Using a similarly constructed rating for reported enjoyment of different television programmes, hard-of-hearing viewers were found to exhibit a small reduction in enjoyment across the majority of programme types. Slater et al. (2010) identify that innovative applications of DTV will be a key enabler for people with disabilities, such as increased and improved subtitling.

\section{The technological possibilities of DTV for subtitling}

Technological facilitation has the potential to realize benefits like those advocated by Jensema et al. (2000), who assessed the time spent viewing subtitles on television programmes. Data tracking eye movement in television viewing was analysed to determine the percentage of time each subject actually looked at the captions on the screen. It was found that subjects gazed at the subtitles 84 per cent of the time, at the video picture 14 per cent of the time and off the video 2 per cent of the time. Age, sex and educational level appeared to have little influence on time spent viewing captions. If the experience of watching television with subtitling is an experience dominated by the subtitle rather than the image, possible innovations in DTV with individual settings and preferences could provide an individually tailored subtitling experience for viewers. Burnham et al. (2008) assessed the roles of subtitle rate and text reduction as factors that appear to affect the comprehension of subtitles by people who are deaf or hard-of-hearing. Reading level emerges as a dominant factor: more proficient readers show better comprehension than poor readers and are better able to benefit 
from caption rate and, to some extent, text reduction modifications. Fixed or universal subtitling may not be beneficial as a means to increase accessibility of television programmes, and the possibility of customized captioning services via DTV may improve the accessibility of television across more of the deaf and hard-of-hearing audience than current services.

Erber and Leigh (2008) support these findings with research into child audiences. Children with impaired hearing (and delayed language development) may not be able to read at a sufficient rate or with sufficient comprehension to understand subtitled film or television dialogue - unless it is specifically tailored to their needs. Tyler et al. (2009) reiterated these findings in investigating the effect of subtitle rate on the comprehension of educational television programmes by deaf school students. The rate of subtitle delivery affected the comprehension of educational programmes by both better- and poorer-reading deaf school children. The research undertaken here will look at subtitling as a critical application of technology within the medium, assessing the following research question (RQ2) with regard to the universality of the importance of subtitling as opposed to the age-specific importance it may have with regard to the age-skewed deaf and hard-of-hearing audience: is subtitling the most important technological affordance available to deaf and hard-of-hearing people when watching television, and is this proportional to the age of the audience?

The use of subtitling is not only a matter of preference, but also statutory concern in the United Kingdom. The Communications Act (2003) requires a target of 80 per cent subtitled programmes for major television stations (BBC, ITV, etc.) but does not make any recommendations beyond the macro-level insistence on provision of service. Ofcom (2006) set out guidelines for the provision of services for deaf and hard-of-hearing audiences with regard to subtitling television programmes. The report addresses quality at the micro level, stating that the aim should be to synchronize speech and subtitling as closely as possible. 
Subtitle appearance should coincide with speech onset, and disappearance should coincide roughly with the end of the corresponding speech segment. In live programmes, the aim should be to keep the inevitable delay in subtitle presentation to the minimum (no more than three seconds) consistent with accurate presentation of what is being said. If possible, subtitles should not over-run shot changes and should commence on a shot change when synchronous with the start of speech (Ofcom 2006). From a statutory perspective, a high quantity of subtitling is expected to be a given, but a high quality of subtitling on programmes is subject to guideline advice. This is despite research reported earlier that argues that subtitle users need to be able both to watch what is going on and to read the subtitles, so it is important that these are as accurate as possible so that viewers do not need to guess what is meant by an inaccurate subtitle. Jellinick (2011) states that the U.K. government is currently involved in a re-examination of rules and regulations for television subtitles and audio-description with the development of new interactive television (iTV) services such as YouView. The research in this article will implicitly assess the impact of the Ofcom guidance through the perceptions of the deaf and hard-of-hearing audience on subtitle quality.

Despite the needs of viewers for quality subtitling, the provision extensions afforded by DTV have not been assessed favourably. The research done by both de Castro et al. (2011) and Rander and Looms (2010) assessed real-time subtitle synchronization in live television programmes. In live subtitling environments, the audio transcription process will deliver the corresponding subtitles several seconds after the speech fragment has been received. The disturbing effect of the resulting lack of synchronism is still a challenging issue in the accessibility to live multimedia and causes dissatisfaction among audiences. A more recent Ofcom (2014b) report reviewing live subtitling quality reveals problems of latency, inaccuracy, poor presentation, and other issues associated with the provision of live 
subtitling. The subtitle provider for the BBC, Channel 4 and Sky is enhancing the software that respeakers and stenographers use and the $\mathrm{BBC}$ has also been searching for suitable technologies in addressing such issues (Ofcom 2014b). Apart from the augmented technological solution provided, it also suggests local buffering for video and audio streams in personal video recorders in order to re-synchronize the subtitles and the video/audio to which they refer, achievable using DTV (Rander and Looms 2010); or a slightly-delayed online streaming of a channel, in which the subtitling provided on the live version is automatically reformatted in blocks to synchronize the visual components (Ofcom 2014b).

Subtitling has been identified as a key technological affordance in the use of television by deaf and hard-of-hearing audiences. Following the switch to DTV in the United Kingdom, the digital platform (with its increased ability for affordances with regard to personalization of subtitling and improved visual performance) should effect a positive change in quality of subtitles. The third research question posed here (RQ3) again probes the universality of this issue: are difficulties reported with subtitling on digital television related to the age of the deaf or hard-of-hearing audience member?

\section{The possibilities and accessibility of DTV as an interactive media technology}

There are also potential issues with the technology - the set-top boxes (STBs), the receiver, the interface or the service - itself that could affect the use of television for this audience post-switchover that derive from the technological complexity that emerges from increased affordance. Maad (2003) argued that research and development in the area of pervasive technologies and in particular iTV has not delivered the aimed objectives of improving accessibility. This was attributed to several factors, including the limitation of the prevalent paradigms of interaction with DTV, the limitation of the DTV technology and the lack of the development of truly interactive and universally accessible multimodal iTV content. Gill and Perera (2003) argue that when universal design is not applied to a 
developing technology such as DTV, it is possible that significant numbers of people will be excluded from accessing or easily using it. Cavender and Ladner (2008) argue that universal solutions (e.g. uniform adoption of DTV) to the issues surrounding inclusivity for deaf and hard-of-hearing audiences are not appropriate - there is a considerable variation in the severity and nature of the disability, and technological solutions must be both diverse and accommodating to address this reality of the disability.

The inclusivity of DTV STBs was explicitly addressed by Keates and Clarkson (2004). This U.K.-based research focussed on the 2004 generation of STBs that provide 'freeto-view' services. Young users were found to experience little difficulty in using the STBs regardless of the extent of their impairment, most probably because of wide experience with high-technology products, whereas older people had the most difficulty. The researchers argued that manufacturers should be encouraged to look beyond the stereotypes of young, severely, impaired people when considering who may have difficulty using their STBs and to also consider the needs of older adults and those who may not be familiar with the interaction paradigms used (including deaf and hard-of-hearing users in the older population). The DTV4all (2011) Joint Recommendations for Future Access Services, Devices and Platforms report argues that all future DTV projects should have feedback mechanisms for any user action or input (e.g. through vibration, sounds, speech) to create a customizable and learnable interface for all users to address these concerns.

In a similar vein, Keith and Whitney (2011) argue that the use of the design for all philosophy at all stages of the process of specification, design and management of information and communication technology systems and products fosters an understanding of user diversity and supports the quality of life of older and disabled people. The design of DTV systems and technology falls within this design remit. Cruickshank et al. (2007) echo this, identifying DTV as having the potential to revolutionize the way we consume broadcast 
media, but identifying that users still find both the notion of DTV and the services currently available problematic in terms of interface, difference from previous services and perceived complexity. Bhachu (2011) summarizes many of the technological issues in DTV, stating that it is full of interactive content that was not previously available through analogue television. However, the interactive content can be complex to use, which in turn creates obstacles for users, and in particular older adults, to access this content. The additional functionality brings with it an increase in complexity and an increase in the physical and mental demands put on the user to operate these functions. This is at a time where the older population is growing throughout modern-day society, and because of age-related decline of physical and mental attributes, this group in particular will find DTV a challenge to use. There is consequently a need to make DTV interactive content more accessible to older adults.

In the UK, Ofcom (2004) commissioned research into supporting the most vulnerable consumers, including deaf and hard-of-hearing audiences, through digital switchover. The key finding from this analysis of the digital adoption process was that those who are likely to find the switch to DTV most difficult are people who have nobody to whom they can turn to for support. That is, digital switchover presents the biggest challenges for those who are socially isolated - people who will have difficulty in finding out about switchover, in understanding what it means and hence who are unable to take effective steps to deal with it. People without an adequate network of support from friends, family, neighbours or carers will therefore be particularly vulnerable. Given the previous research, deaf and hard-ofhearing individuals were particularly likely to be part of this group.

Despite these concerns, Carmichael et al. (2005) identified that the switchover to DTV would bring about an expansion of entertainment and information services available via television and in doing so fundamentally change traditional concepts of household viewing. The researchers argued that from a U.K. perspective, the technological infrastructure of DTV 
has the potential to foster the social inclusion of vulnerable groups, such as disabled and elderly people, by improving their ability to receive enhanced access to content and other services available via this expanding medium. Yet, despite encouragement from a variety of stakeholders including Ofcom that recognizes this opportunity, a significant risk of increasing their marginalization and disfranchisement was identified. This is partly due to the fact that DTV equipment is inherently more complex to operate than analogue, but more importantly steps have not been taken to ensure that consideration is given to the wide diversity of abilities within the viewing population for the design of DTV equipment and services. While the move to digital switchover increases content and introduces interactive services available through the television, key aspects of usability and accessibility had been overlooked by those responsible for encouraging this new infrastructure's inclusive development.

Such disconnections between affordance and use resonate with Goggin and Newell's (2007) identification of a paradox in disability and inclusive information technology. Given that there is now greater knowledge about disability and design, the inability to develop accessible and inclusive technology becomes a key question, specifically, whether it is because inclusive technology is not profitable, and unattractive for businesses and unsustainable as an industry. The power relations of disability and the crucial role played by disability's cultural and social constitution are critical to this consideration; inclusivity and accessibility (especially in the context of switchover) must be considered in the light of the wider economic issues concerning switchover.

Carey (2005) offers a short summary of what needs to be done to enhance accessibility using digital media. First, there needs to be a concentration on digital data design and creation to see that it accords with basic rules and accessibility principles. Second, programmable user interfaces require development to enable individualized accessibility needs to be met. Third, cable-free user interfaces, such as screens and keyboards, can be used 
to provide an optimal user environment. Finally, data provision systems that respond to user behaviour can be used to increase the tailoring of services to specific users. Carey argues that these proposed solutions are all affordable technological innovations that can be used, rather than having uniform operating systems and environments. As DTV is both a broadcast and digital platform, the effect of its implementation can be gauged against these concerns. The final research question (RQ4) will assess the use of further interactive features provided by DTV: is there an age difference in the use of interactive features on DTV platforms? In doing this, there are a number of issues that can be assessed: the levels of support offered to vulnerable audience members in using a critical technology for social inclusion, the awareness of the services provided and whether a digital divide in the use of digital communications has been partially bridged through the introduction of DTV.

\section{Findings of the DTV and deaf and hard-of-hearing audiences in Wales study}

The aim of this research was to identify the patterns of DTV adoption and usage within the deaf and hard-of-hearing audience in Wales and evaluate the current status of DTV accessibility after the switchover. We focus on one particular vulnerable social group, the deaf and hard-of-hearing community in Wales, evaluating the enabling and disabling effects of DTV.

An easy-to-follow questionnaire was developed following this pilot study. The questionnaire was designed to extract both quantitative data and qualitative data from respondents. ${ }^{1}$ The questionnaire consisted of four sections investigating the following areas: (1) demographics, (2) media usage: preference and accessibility, (3) DTV usage: preference and accessibility and (4) suggestions on improving DTV service. A total of 900 paper copies of questionnaires were distributed and disseminated to target audiences who are deaf or hardof-hearing across Wales, along with information on completing the survey online if the participant preferred this method, and this resulted in 240 complete questionnaires returned. ${ }^{2}$ 


\section{Demographics of the sample of participants}

[Figure 1 Here]

Figure 1 indicates that there is a skewed distribution of ages of participants in the survey, with 62 per cent of the sample population over the age of 65 years, reflecting the fact that hearing loss affects senior citizens more often. This is to be expected given that the sampling was of persons specifically identified as having a difficulty with hearing, which is more prevalent in older people in the general population. This is in accordance with the Action on Hearing Loss (2011) figure showing that 65 per cent of people with hearing loss are of retirement age.

With regard to degree of hearing loss, respondents were allowed to respond to as many options as appropriate regarding their deafness and hearing loss (Table 1).

Degree of hearing loss

respondents

Wear digital hearing aid 162

Hard-of-hearing 116

Have tinnitus

Deaf

Lip read

Have balance problems

Have Ménière's disease

Use British Sign Language (BSL)

Wear hearing aid, not sure if digital 
Deafened

Use Sign Supported English (SSE)

Wear non-digital hearing aid

Wear a bone-anchored hearing aid

\section{2}

7

2

1
0.5

\section{Table 1: Degree of hearing loss.}

The key findings were that most respondents, over two-thirds (68 per cent), have digital hearing aids and a further 8 per cent of the respondents wear other types of hearing aids; nearly half of the respondents (48 per cent) identified themselves as hard-of-hearing and one-third as deaf (33 per cent); 8 per cent of respondents reported using BSL and a further 3 per cent of respondents use SSE, which indicates that questions around the use of BSL and BSL-signed programmes would be answered by few of the respondents in the survey.

\section{Results on the Research Questions}

Research question 1: is television used more by older deaf and hard-of-hearing audiences or younger people of the same group?

\section{[Figure 2 Here]}

The reported data on time spent watching television per day shows that television plays a central role in informing, educating and entertaining deaf and hard-of-hearing audiences in Wales, with 96 per cent of the respondents watching more than one hour of television every day. More than half of the respondents (58 per cent) watch two to five hours television per day. One-third of the respondents (33 per cent) watch more than four hours of television per day (Figure 2). The average hard-of-hearing audience watches 3.39 hours television per day. This figure is largely compatible with the Broadcasters Audience Research Board statistics from the same period (August 2013). These statistics suggest that the average U.K. audience spends 24.49 hours per week watching television, that is 3.49 hours per day watching television (Broadcasters Audience Research Board 2013). 
A chi-square test of independence was performed to examine the relation between age and hours spent watching television. The relation between these variables was not found to be significant, $X^{2}(56, N=240)=56.58, \mathrm{p}(0.443)>0.05$. Older people did not use DTV significantly more than younger people in the sample group, indicating that DTV has an important role to play (given the daily amount of television watched) in the lives of all of the audience members sampled.

Research question 2: is subtitling the most important technological affordance available to deaf and hard-of-hearing people when watching television, and is this proportional to the age of the audience?

An important subset of the data was on the comparison of subtitles and BSL in facilitating television viewing as the data illustrates the critical importance of subtitling to the audience.

[Figure 3 Here]

Comparing the data on the use of subtitles and the use of BSL, we found that subtitles are the most popular solution to hearing difficulties when watching television. These findings reflect the fact that subtitling is much more prevalent, with the main broadcasters (BBC, ITV, Channel 4 and Five) all subtitling upwards of 80 per cent of their content, while BSL programmes are confined to off-peak hours; 89 per cent of respondents reported using subtitles at some point and 54 per cent all the time to watch television (Figure 3 ). The focus in this research question on the use of subtitles and perceived difficulties in their usage is therefore particularly relevant. The vast majority of respondents do not use BSL programming frequently, and only 24 reported using BSL programming more than rarely (10 per cent).

A chi-square test of independence was performed to examine the relation between age and use of subtitles. The relation between these variables was not found to be significant, $X^{2}$ 
$(28, N=240)=35.65, \mathrm{p}(0.152)>0.05$. Older people do not use subtitles significantly more than younger people in the sample group, indicating that subtitles are critically important as an accessibility technology to the majority of the audience members sampled.

In general, respondents enjoy the better picture quality, variety of programmes and better sound quality available on DTV since the digital switchover completed in Wales; 62.5 per cent of the respondents believe that DTV provides a better service than the old analogue television (Table 2).

Perceived benefits of DTV Number of Percentage respondents

Better picture quality 148 62

Better range and quality of programmes 115 48

Better sound quality 98

Better subtitling options 84 35

Better quality of subtitles 78

Other 13 5

Better sign language availability 5 2

Table 2: Perceived benefits of switching to digital television by respondents.

The improvement of subtitling and subtitling options is important (35 per cent and 33 per cent, respectively). To test whether this is also related to the age of the participants, chisquare tests of independence were used. The relation between age and having the opinion that DTV offered better subtitling options was not found to be significant, $X^{2}(7, N=240)=$ 13.03, $\mathrm{p}(0.071)>0.05$. The relation between age and having the opinion that DTV offered better quality of subtitling was also not found to be significant, $X^{2}(7, N=240)=8.84$, p $(0.265)>0.05$. Older people did not significantly assess subtitle quality as improved 
compared to younger people in the sample. This finding again emphasizes the universality in subtitling for the experience of DTV.

Research question 3: are difficulties reported with subtitling on DTV related to the age of the deaf or hard-of-hearing audience member?

The improvement in subtitling noted earlier does sit in contrast with the reported difficulties of using DTV (Table 3).

Reported difficulties of using DTV

Number of Percentage

respondents

Delay on subtitles

148

62

Subtitles missing

140

58

Difficult to hear speech

125

Misspelling on subtitles

121

Subtitles use wrong words/inappropriate

108

words

Background noise

104

Poor-quality subtitles during live broadcast

101

Subtitles moving too quickly

66

Poor sound quality

Other

Sparse subtitles

Cannot follow subtitles

Cannot get subtitles to work

Not enough sign language availability

Cannot access subtitles due to low signal 
strength

Sign language programmes at inconvenient

times

Size of subtitles too small

Table 3: Reported difficulties of using digital television.

More than half of the respondents reported difficulties in the delayed subtitles, missing subtitles, misspelling on subtitles and difficulties hearing speech. Inappropriate linguistic choices in subtitles (45 per cent), background noise (43 per cent) and poor subtitles during live broadcasts ( 42 per cent) all indicate that for this demographic (that use subtitles often) the service provided is often perceived as less than adequate. As stated earlier, the Communications Act (2003) ensures the provision of subtitles, but the quality of subtitles is subject to guidance, not statute; these figures indicate that the quality of subtitles is a major issue post-digital switchover.

To assess whether the issues identified with subtitling here are subject to age effects, again a series of chi-square tests of independence were performed with factors that were identified as being very important in difficulties in watching DTV, that is those factors identified by more than 30 per cent of the sample (Table 4).

\begin{tabular}{llll}
$\begin{array}{l}\text { Issue with subtitling } \\
\text { identified in relation to }\end{array}$ & $\begin{array}{l}\text { Degrees of freedom } \\
\text { and number of }\end{array}$ & Chi-square value & Significance \\
age & participants & & \\
\hline Perceived delay of & $7 ; 240$ & 9.40 & $\mathrm{p}(0.225)>0.05$ \\
subtitles & & 6.60 & $\mathrm{p}(0.471)>0.05$ \\
Reporting subtitles to & $7 ; 240$ & & \\
be missing & &
\end{tabular}




\begin{tabular}{llll}
\hline Reporting misspelling & $7 ; 240$ & $\mathrm{p}(0.6679)>0.05$
\end{tabular}

of subtitles

Subtitles having wrong $7 ; 24$

$\mathrm{p}(0.261)>0.05$

or inappropriate words

Reporting poor quality $\quad 7 ; 240$

9.11

$\mathrm{p}(0.245)>0.05$

of subtitles in live

broadcasts

Subtitles moving too

7; 240

6.60

$\mathrm{p}(0.471)>0.05$

quickly

Reporting sparse

$7 ; 240$

20.85

$\mathrm{P}(0.004)<0.05$

subtitles

Table 4: Results of chi-square tests of independence with regard to the relation between age and subtitling difficulties.

With the exception of reported problems with sparse subtitling, significant differences were not found across age groups with regard to problems with subtitling. This finding again emphasizes the universality in subtitling for the experience of DTV.

Another key issue from the results is sound quality. Chi-square tests of independence were performed to examine the relations between age and difficulties in hearing speech, reported difficulties with background noise and poor sound quality. The relation between age and difficulty in hearing speech was found to be significant, $X^{2}(7, N=240)=24.10$, p $(0.001)<0.05$. The relation between age and difficulty with background noise was found to be significant, $X^{2}(7, N=240)=20.08, \mathrm{p}(0.005)<0.05$. The relation between age and reported poor sound quality was found to not be significant, $X^{2}(7, N=240)=4.92$, p $(0.669)$ $>0.05$. These findings are significant as they illustrate that while subtitle difficulties are relatively universal across the sample, there are clear age differences with auditory issues. In particular, hearing speech is significantly related to age. While these findings may be 
expected with regard to the sample group, the finding that older people report significantly greater problems with speech and background noise is important with regard to the composition and editing of programmes and their accessibility.

Research question 4: is there an age difference in the use of interactive features on DTV platforms?

In assessing the possible existence of a digital divide between older and younger users of DTV, a platform-specific analysis of the technological methods used to watch television can give some initial scope to the question (Table 5).

Technology used to watch television

Television - on air

Television - watch again via iPlayer and Clic

Television - watch again via on-demand services such as

Virgin On Demand and YouView

Television - recorded programmes stored on a PVR, hard

disk recorded or DVD

Television - via service such as Netflix or LoveFilm

Television - via social media such as YouTube
The number of Percentage

respondents

Table 5: Technology used in watching television.

Overwhelmingly, respondents used a dedicated television set that receives digital broadcasts to watch television (96 per cent). Although online services and digital recording are used increasingly, they are not yet as popular as broadcast television.

Worth noting here is also the growing popularity of the Internet broadcasting service and software application iPlayer and Clic developed by the BBC and S4C. Almost one-third 
of the respondents use these services. These digital platforms enable viewers to watch live television or catch up, as well as enable viewers to watch television via various digital devices such as an iPad. Deaf and hard-of-hearing audiences are part of the trend in which the traditional pattern of television viewing as a communal activity among family members is evolving into a more personalized viewing experience.

[Figure 4 here]

Although the general awareness of video-on-demand (VoD) services and 'red button' for extra information is stronger, it seems that only a small proportion of respondents actually used these services regularly ( 8 per cent and 14 per cent, respectively) (Figure 4)..

To assess whether the use of interactive services on DTV is subject to age effects, again a series of chi-square tests of independence were performed (Table 6).

Use of interactive Degrees of freedom Chi-square value Significance service on DTV related and number of to age participants

\begin{tabular}{lll}
\hline Alter colour and size of $28 ; 240$ & p $(0.022)<0.958$
\end{tabular}
subtitles

Use of $\mathrm{VoD}$

$$
\mathrm{p}(0.000)<0.05
$$

Use of red button

$14 ; 240$

$$
\mathrm{p}(0.000)<0.05
$$

Table 6: Results of chi-square tests of independence with regard to the relation between age and use of interactive services.

These results show that there are significant differences in the knowledge and use of interactive services provided by DTV. With regard to changing subtitles, use and awareness is low for all groups but there is a significant age effect with regard to knowledge despite this low uptake. With regard to VoD and red button (interactive) services, there are significant differences in both knowledge and use of these services, offering a clear illustration of the digital divide between users of DTV who are deaf or hard-of-hearing. 


\section{Conclusions}

Broadcast television occupies a central position in the media consumption of this community, with 96 per cent of respondents using the medium and more than half of the respondents (57 per cent) watching two to five hours of television per day. Public broadcasters (especially the BBC) are regarded as the major sources of information. This study has established that subtitles are the most important facilitating tool for deaf and hardof-hearing audiences in their reception of television messages. Common problems associated with sound quality and subtitles affect all members of the deaf and hard-of-hearing audience. A significant amount of this sample relies on digital hearing aids (68 per cent) and also lip reading (about one-third), and so the demand for better sound quality and a better subtitling service is acute. The universality of problems with subtitles is particularly an issue, as the dependence on this service is so widespread and there are a number of statutory guidelines on subtitling that emphasize the importance of accuracy in this service. Yet this research can conclude not only that issues with subtitling remain a considerable barrier to television use for the deaf and hard-of-hearing audience, but also that this barrier transcends notions of a 'digital divide' and affects the viewing experience across the audience irrespective of age. Familiarity with digital technology and interactive services does not mediate the effect of this fundamental barrier to accessing the basic services of television.

While this research has identified a number of barriers facing people who are deaf and hard-of-hearing in accessing information, education and entertainment via DTV, most significantly there is a major divide between older and younger members of this sample on both knowledge and use of interactive services. The affordances of DTV to make television more accessible to the deaf and hard-of-hearing audience are present but are not currently sufficiently utilized to bridge the gap between audience and content. This, it can be concluded, means that the advantages of access to television summarized in this article are 
not being efficiently met through the current platform. Training and education programmes on the under-utilized services on the DTV platform could help narrow this gap, but crucially an improvement of the critical access services is a priority following this research. This finding indicates that there needs to be greater efforts made both to improve information on these services, particularly aimed at older audience members, and to address accessibility of these services for older people. While it is tempting to advocate training as a fix-all solution, the platform itself must play a role in this process. Improving the interface to account for the needs of older people with hearing difficulties, involving older people and advocacy groups to participate in design consultation and implementing a thorough testing and feedback-led development are some of the measures that could assist in reducing this barrier to interactive content, which in itself may improve the viewing experience. The most important finding of this research is through the reliance on subtitling for this group, and the universal nature of issues with quality identified across age groups. While policy and regulatory bodies have clearly dealt with issues around the quantity of subtitles available, the quality of subtitling remains a critical barrier to understanding programming and enjoying television services for deaf and hard-of-hearing audience members. A greater priority for the quality of subtitling provided must be the next focus of policy-makers. In the United Kingdom, this might take the form of greater attention to quality by the regulator (Ofcom), but more innovative and powerful interventions in the form of statutory requirements laid down by government or through extended requirements as part of the $\mathrm{BBC}$ charter review might be necessary to ensure improved quality.

Future research may assess gender issues within the barriers to access and may look to extend the research longitudinally across changes in service provision. Differences in DTV platforms may also be significant, and this could be investigated further. A technological study on the production of subtitles may also illustrate the difficulties involved with the 
provision of this service and could be critical in a discourse with this research on contextualizing the importance of subtitles and understanding how to improve the service. A longitudinal study on the use of interactive services, with an element of public education or training, may also capitalize on these findings and could bring practical benefits from this research.

\section{Acknowledgements}

This project received financial support from Action on Hearing Loss Cymru, BBC Cymru Wales, Bridging the Gaps Fund (EPSRC Swansea University), Coleg Cymraeg Cenedlaethol and the Research Institute of Arts and Humanities (Swansea University). This article was completed with additional support from funding from the European Research Council as part of the Programmable City project at Maynooth University (grant: ERC-2012AdG 323636-SOFTCITY).

\section{References}

Action on Hearing Loss (2011), 'Facts and figures on hearing loss and tinnitus', http://www.actiononhearingloss.org.uk/your-hearing/about-deafness-andhearingloss/statistics/ /media/56697A2C7BE349618D336B41A12B85E3.ashx. Accessed 20 May 2014.

Arlinger, S. (2003), 'Negative consequences of uncorrected hearing loss - A review', lnternational Journal of Audiology, 42:2, pp. S17-21.

Austin, B. A. (1980), 'The deaf audience for television', Journal of Communication, 30: 2, pp. 25-30.

Austin, B. A. and Myers, J. W. (1984), 'Hearing-impaired viewers of prime-time television', Journal of Communication, 34: 4, pp. 60-71. 
Bhachu, A. S. (2011), 'Inclusive technologies for enhancing the accessibility of digital television', SIGACCESS Newsletter, 99, pp. 2009-12.

Broadcasters Audience Research Board (2013), 'Total viewing summary - Monthly', http://www.barb.co.uk/viewing/monthly-total-viewingsummary?period\%5B\%5D=20130801\&button_submit=View+figures. Accessed 22 May 2014.

Brooks, M. (2006), 'Challenges for warning populations with sensory disabilities', B. Van de Walle and M. Turoff (eds), Proceedings of the 3rd International ISCRAM Conference, Newark, NJ, May.

Burnham, D., Leigh, G., Noble, W., Jones, C., Tyler, M., Grebennikov, L. and Varley, A. (2008), 'Parameters in television captioning for deaf and hard of hearing adults: Effects of caption rate vs. text reduction on comprehension', Journal of Deaf Studies and Deaf Education, 13:3, pp. 65-77.

Carey K. (2005), 'Accessibility: The current situation and new directions', Ariadne, 44, June. http://www.ariadne.ac.uk/issue44/carey/. Accessed 7 October 2011.

Carmichael, A., Rice, M. and Sloan, D. (2005), 'Digital interactive television in the UK: Is the opportunity for “inclusivity” being missed?' Proceedings of Accessible Design in the Digital World Conference, Dundee, May.

Cavender, A. C. and Ladner, R. E. (2008), 'Hearing impairments', chapter 3 in Harper, S. and Yesilada, Y. (eds), Web Accessibility: A Foundation for Research, New York: Springer, pp. 25-36.

Chia, E. M., Wang, J. J., Rochtchina, E., Cumming, R. R., Newall, P. and Mitchell, P. (2007), 'Hearing impairment and health-related quality of life: The Blue Mountains Hearing Study', Ear and Hearing, 28:2, pp. 187-95. 
Communications Act (2003), 'UK government', http://www.legislation.gov.uk/ukpga/2003/21/contents. Accessed 21 March 2014.

Cruickshank, L., Tsekleves, E., Whitham, R., Hill, A. and Kondo, K. (2007), 'Making interactive TV easier to use: Interface design for a second screen approach', The Design Journal, 10:3, pp. 41-53.

de Castro, M., Carrero, D., Puente, L. and Ruiz, B. (2011), 'Real-time subtitle synchronization in live television programs', Broadband Multimedia Systems and Broadcasting (BMSB), 2011 IEEE International Symposium on, pp.1-6, 8-10, June 2011.

Digital UK (2006), Press Release (24/02/2006) 'Digital UK heralds 'golden age of TV', available at: http://www.digitaluk.co.uk/_data/assets/pdf_file/0004/19597/240206_vlv_conferenc e.pdf.

DigitalUK (2012), 'Digital TV switchover 2008-2012 final report', http://www.digitaluk.co.uk/_data/assets/pdf_file/0019/82324/DigitalUK_Switchover final_report_Nov2012.pdf. Accessed 20 May 2014.

DTV4all (2011), 'Joint recommendations for future access services, devices and platforms', http://www.psp-dtv4all.org/. Accessed 4 October 2011.

Erber, N. and Leigh, G. (2008), Submission to the Investigation into Access to Electronic Media for the Hearing and Vision Impaired. London: Royal institute for Deaf and Blind Children.

Gibson, J. J. (1977), 'The theory of affordances', in Robert Shaw and John Bransford (eds), Perceiving, Acting, and Knowing, Hoboken, NJ: John Wiley \& Sons, pp. 127-43. 
Gill, J. M. and Perera, S. A. (2003), 'Accessible universal design of interactive digital television', 1st European Interactive Television Conference, Brighton, pp. 83-89.

Goggin, G. and Newell, C. J. (2007), 'The business of digital disability', The Information Society, 23: 3, pp. 159-68.

Iosifidls, P. (August 2005), 'Digital switchover and the role of the new BBC services in digital television take-up', Convergence, 11: 3, pp. 57-74.

_ (2007), 'Digital TV, digital switchover and public service broadcasting in Europe', Javnost - The Public: Journal of the European Institute for Communication and Culture, 14: 1, pp. 5-20.

Jellinick, D. (2011), 'UK government refreshes e-accessibility policy’, E-Government Bulletin Live, http://www.headstar.com/site/scripts/news_article.php?newsID=195 . Accessed 5 October 2011.

Jensema, C. J., Danturthi, S. and Burch, R. (2000), 'Time spent viewing captions on television programs', American Annals of the Deaf, 145: 5, pp. 464-68.

Keates, S. and Clarkson, P.J. (2004), Accessing the Accessibility of Digital Television SetTop Boxes. In Designing a More Inclusive World, edited by Keates, S., Clarkson, J., Langdon, P. and Robinson, P., pp. 183-192. London: Springer.

Keith, S. and Whitney, G. (2011), 'Mainstreaming accessibility? Multidisciplinary problems or technical solutions', International Journal of Knowledge and Web Intelligence, 2: 1, pp. 53-63.

Lochrie, M. and Coulton, P. (2012), 'Sharing the viewing experience through second screens', EuroiTV'12 Proceedings of the 10th European Conference on Interactive TV and Video. NY: ACM, pp. 199-202. 
Maad, S. (2003). 'The potential and pitfall of interactive TV technology: An empirical study', Paper presented at the International Conference on 'Television in Transition', held at MIT, 2-4 May 2003.

Norman, D. (2013), The Design of Everyday Things (revised and expanded ed.), New York: Basic Books, p. 11.

Ofcom (2004), 'Supporting the most vulnerable consumers through digital switchover', http://www.digitaltelevision.gov.uk/publications/pub_cp_dso_report.html. Accessed 4 October 2011.

(2006), 'Guidelines on the provision of television access services', http://stakeholders.ofcom.org.uk/broadcasting/guidance/otherguidance/tv_access_serv/guidelines/.. Accessed 4 October 2011, pp. 27-33. (2014), 'Ofcom communication market report', http://stakeholders.ofcom.org.uk/market-data-research/marketdata/communications-market-reports/cmr14/.Accessed 1 March 2015 (2014b), 'Television Access Services 2013',

http://stakeholders.ofcom.org.uk/binaries/research/tv-research/access-servicereports/Access_services_report_2013_for_publication-_Final.pdf. Accessed 1 March 2015.

Rander, A. and Looms, P. O. (2010), 'The accessibility of television news with live subtitling on digital television', Proceedings of the 8th International Interactive Conference on Interactive TV\&Video EuroiTV, 10:3, pp. 155. New York: ACM Press, http://portal.acm.org/citation.cfm?doid=1809777.1809809. Accessed 14 October 2011.

Sancho-Aldridge, J. and Davis, A. C. (1993), 'The impact of hearing impairment on television viewing in UK', British Journal of Audiology, 27 (3), pp. 163-73. 
Slater, J., Lindström, J.-I. and Astbrink, G. (2010), 'TV and broadband: Innovative applications for people with disabilities', Telecommunications Journal of Australia, 60: 2, pp. 20.1-20.14. Monash University ePress.

Smith, P. and Steemers, J. (2007), 'BBC to the rescue!: Digital switchover and the reinvention of public service broadcasting in Britain', Javnost - The Public: Journal of the European Institute for Communication and Culture, 14: 1, pp. 39-55.

Starks, M. (2007), Switching to Digital Television: UK Public Policy and the Market, Bristol: Intellect Books.

Tyler, M. D., Jones, C., Grebennikov, L., Leigh, G., Noble, W. and Burnham, D. (2009), 'Effect of caption rate on the comprehension of educational television programmes by deaf school students', Deafness and Education International, 11: 3, pp. 152-62.

van Dijk, J.A.G.M., (2005), The Deepening Divide, Inequality in the Information Society. London: Sage Publications.

Wales Mental Health in Primary Care (n.d.), 'Information sheet 14: Mental health, deafness and hearing loss', http://www.wamhinpc.org.uk/sites/default/files/information-sheet-14.pdf. Accessed 11 October 2013.

Welsh Government (2013). 'Welsh health survey 2012', http://www.bhfactive.org.uk/userfiles/Documents/welsh health survey 2012.p df. Accessed 20 May 2014 .

\section{Contributor details}

Leighton Evans is a postdoctoral researcher on the ERC-funded Programmable City Project, based at the National Institute for Regional and Spatial Analysis at Maynooth University, 
Ireland. Leighton's primary research interests are in the philosophy of technology and

phenomenology.

Contact: leighton.evans@ nuim.ie

Yan Wu is Senior Lecturer in media studies at Swansea University. Her research interests are in international and comparative journalism, and the transformative effects of information technologies on journalism as a profession.

Contact: y.wu@swansea.ac.uk

Elain Price is Lecturer in media studies at Swansea University. Her research interests are in the history of broadcasting, the history of S4C, Film and Wales, Welsh Television and Welsh Animation.

Contact: e.price@swansea.ac.uk

\footnotetext{
1 The quantitative questions measured facts (e.g. demographics), preferences (e.g. digital television over analogue television) and behaviour (e.g. hours spent in watching television) in the format of nominal or ordinal questions. The qualitative questions measured attitudes (e.g. suggestions to the public broadcasters regarding improving service). The questionnaire was available in English and Welsh, enabling respondents to respond in their preferred language. The questionnaire was available in hard copy as well as via the Swansea University website. A freepost envelope was provided to ensure questionnaires could be returned easily and efficiently. ${ }^{2}$ A total of 339 questionnaires were returned, with 240 valid answers and 99 incomplete, giving a 26.6 per cent response rate. Among the valid questionnaires, 15 responses were completed online and 225 were from a paperbased survey. Among the 99 incomplete questionnaires, 26 were online and 73 from paper copies. All responses were entered into an Excel spreadsheet for quantitative analysis. Percentages in the tables presented in the report may not equal 100 per cent due to respondents selecting multiple answers. For data validation reasons, incomplete surveys are not included in the quantitative analysis.
} 


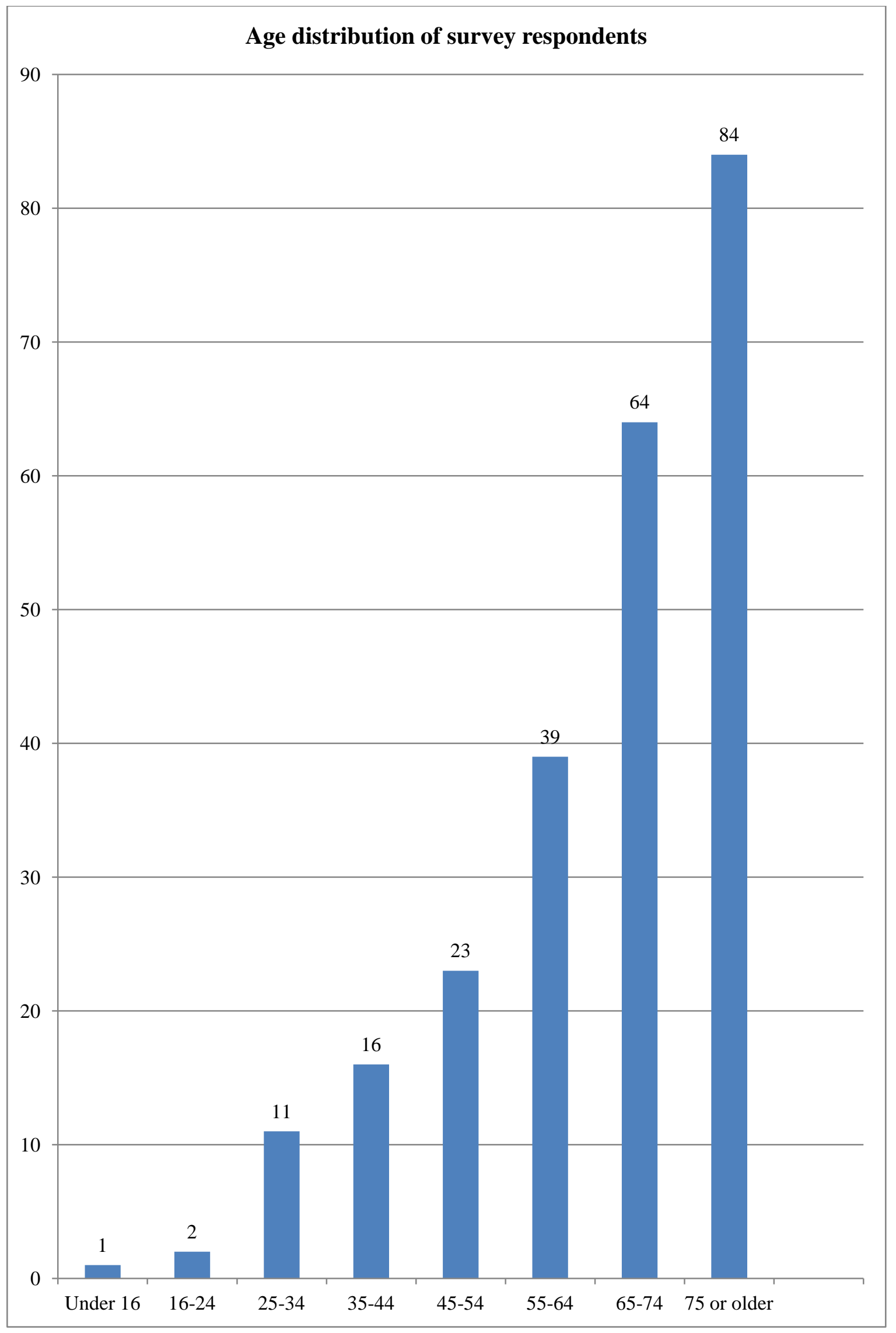

Figure 1 Graphical summary of age distribution of participants 


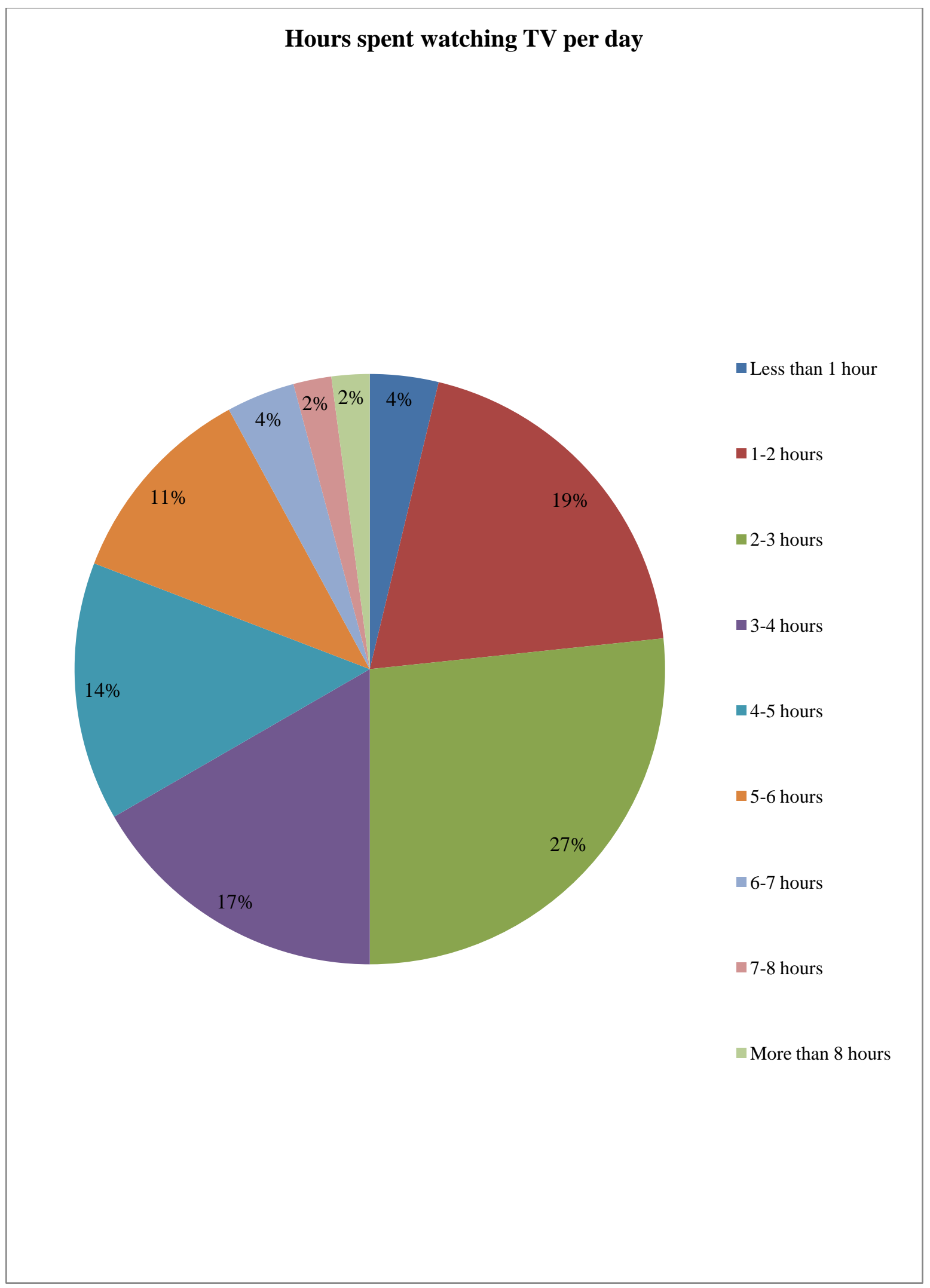

Figure 2 Television Viewing Pattern: Hours spent on watching television daily 


\section{Frequency of using subtitles or BSL in television viewing}

90

80

70

60

50

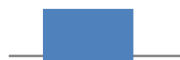

40

20

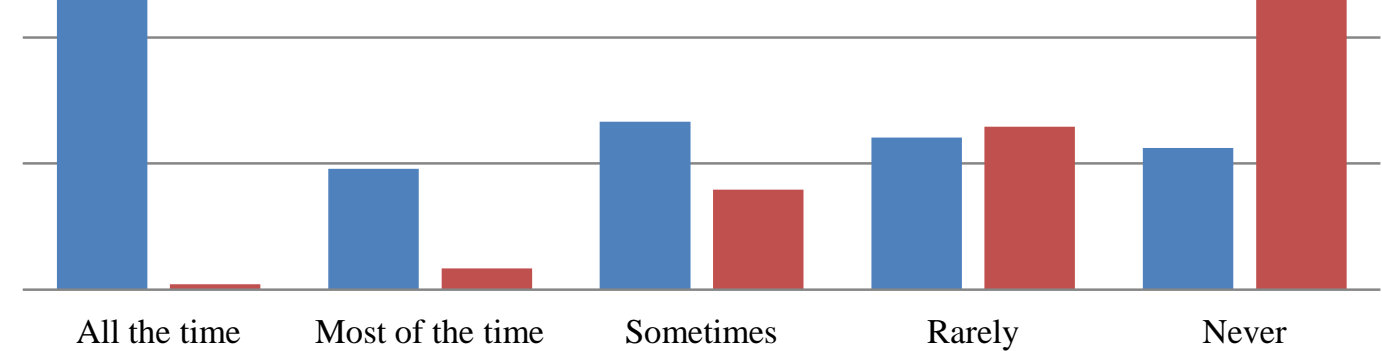

- Subtitles (Percentage \% of respondents)

BSL (Percentage \% of respondents)

Figure 3 Frequency of using subtitles or BSL in television viewing 


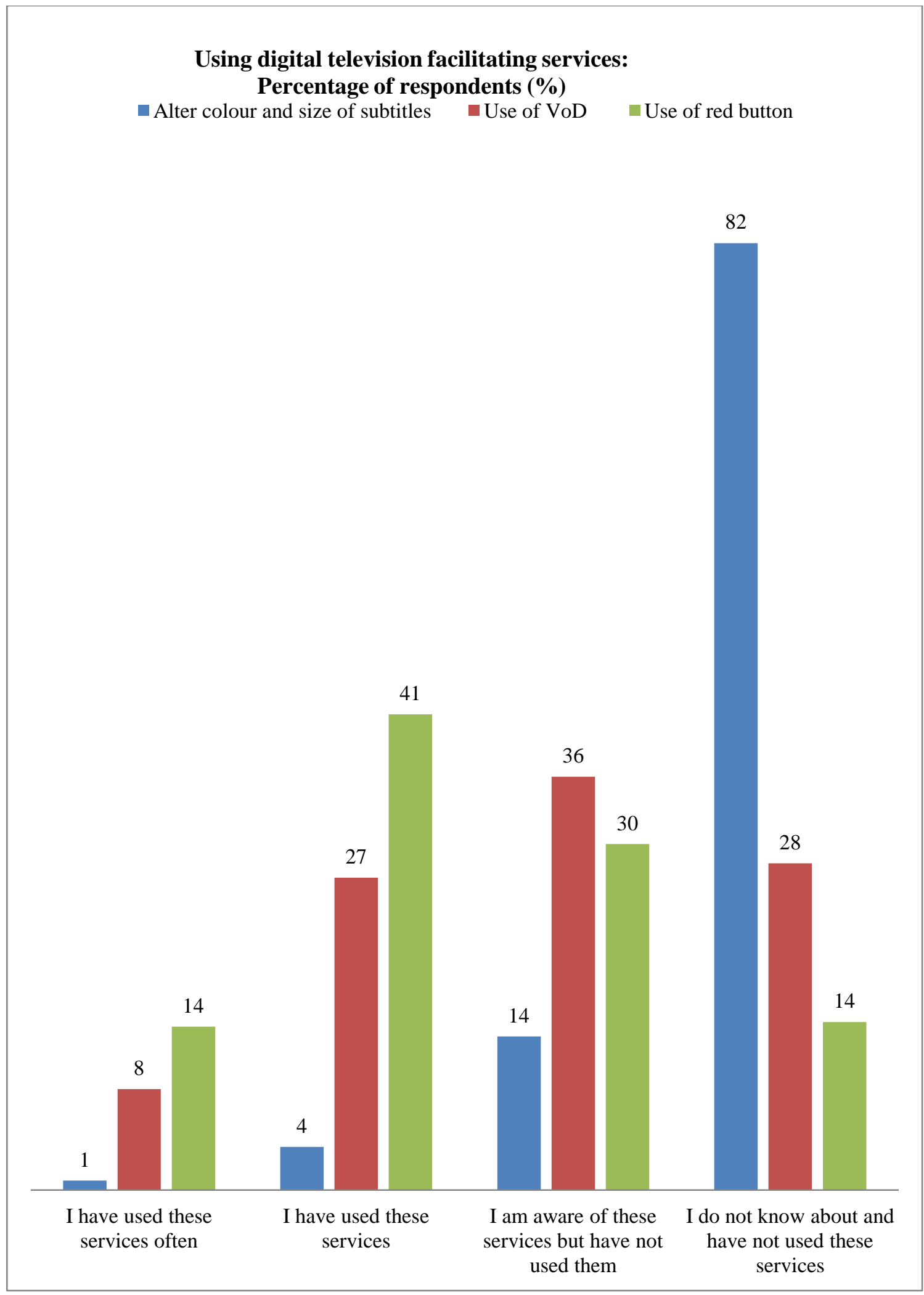

Figure 4 Using digital television facilitating services: A comparison of the use of service to alter colour and size of subtitles (available on a small number of television sets), the use of VoD and the use of red button 